\title{
Pulse profiles and timing of PSR J1757-2421
}

\author{
J. P. Yuan, ${ }^{1,2 \star}$ R. N. Manchester, ${ }^{3}$ N. Wang,,${ }^{1,2}$ J. B. Wang, ${ }^{1}$ X. Zhou, ${ }^{1}$ \\ W. M. Yan, ${ }^{1}$ and Z. Y. Liu, ${ }^{1}$ \\ ${ }^{1}$ Xinjiang Astronomical Observatory, CAS, 150 Science 1-Street, Urumqi, Xinjiang, China, 830011 \\ ${ }^{2}$ Key Laboratory of Radio Astronomy, Chinese Academy of Science, Nanjing, China, 210008 \\ ${ }^{3}$ CSIRO Astronomy and Space Science, Australia Telescope National Facility, PO Box 76, Epping, NSW 1710, Australia
}

Accepted ... Received ...; in original form 2016 Sept.

\begin{abstract}
Pulse arrival time measurements over fourteen years with the Nanshan 25-m and Parkes 64-m radio telescopes have been used to determine the average profile and timing properties for PSR J1757-2421 (B1754-24). Analysis of the radio profile data shows a large variation of spectral index across the profile and an unusual increase in component separation with increasing frequency. The timing observations show that PSR B1754-24 underwent a large glitch with a fractional increase in spin frequency of $\Delta \nu_{\mathrm{g}} / \nu \sim 7.8 \times 10^{-6}$ in May 2011. At the time of the glitch there was a large permanent jump in the rotational frequency accompanied by two exponential recovery terms with timescales of 15(6) and 97(15) days, respectively.
\end{abstract}

Key words: stars: neutron - pulsars: general - pulsars: individual (PSR J1757-2421, PSR B1754-24)

\section{INTRODUCTION}

Pulsars are rapidly rotating and strongly magnetised neutron stars that radiate beams tied to the rotating star through their magnetic fields. Frequent timing observations over months and years can give an accurate model for the spin behaviour of the neutron star. Although pulsars are generally precise clocks, the timing observations have detected the two types of instability of pulsar rotation, glitches and timing noise. A glitch is characterised by abrupt increase in the rotation frequency of pulsar, usually accompanied by an increase in the spin-down rate. This event is often followed by an exponential recovery towards the extrapolation of the pre-glitch behaviour. Up to now, more than 400 glitch events have been detected in at least 140 pulsars, with more than 200 having been published since $2010^{1}$. Observed fractional glitch sizes range from $\sim 10^{-10}$ to $\sim 10^{-5}$ with a bimodal distribution, where the peaks lie around $2 \times 10^{-9}$ and $10^{-6}$ respectively (Yuan et al. 2010a; Espinoza et al. 2011; Yu et al. 2013).

Since the phenomenon was first detected in the Vela pulsar (Radhakrishnan \& Manchester 1969), there has been

\footnotetext{
* E-mail: yuanjp@xao.ac.cn

1 http://www.atnf.csiro.au/people/pulsar/psrcat/(version 1.54, Manchester et al 2005); http://www.jb.man.ac.uk/pulsar/ glitches.html
}

a lot of effort to uncover the glitch mechanism, nevertheless, it is not fully understood. The early studies suggested that glitches result from crustquakes in the neutron star (e.g., Baym \& Pines 1971). When the starquake model proved unviable, it was suggested that glitches are triggered by decoupling and recoupling of vortices between the crust and inner superfluid, resulting in the transfer of angular momentum to the crust (e.g., Anderson \& Itoh 1975; Alpar et al. 1981). Glampedakis \& Andersson (2009) suggested that an r-mode instability may trigger a global unpinning of vortices leading to a glitch. The vortex unpinning model can explain the various aspects of large glitches and post-glitch behaviour (e.g., Haskell \& Antonopoulou 2014), but Zhou et al. (2014) have revived the starquake model, suggesting that material is transported from the equator to the pole, causing the star spin up, with large jumps of $\dot{\nu} / \nu \sim 10^{-6}$ possible.

Timing noise is a continuous, erratic fluctuation of the observed pulsar period, usually modelled as a red noise process in the timing residuals with a correlation on time-scales of months to years. Timing noise may result from a random walk in the pulse phase, spin or spin down, which would give power-law spectra for the timing residuals $\left(S(f) \propto f^{\alpha}\right)$ with spectral indices $\alpha=-2,-4$ or -6 respectively (Boynton et al. 1972). But there is some argument that it cannot simply be modelled as a random walk (e.g., Cordes \& Downs 1985; Hobbs et al. 2010). It may be generated by various intrin- 
Table 1. Details of observations for PSR J1757-2421

\begin{tabular}{|c|c|c|c|c|c|c|c|}
\hline Telescope & Frontend & $\begin{array}{l}\text { Central freq. } \\
\qquad(\mathrm{MHz})\end{array}$ & $\begin{array}{r}\text { Bandwidth } \\
(\mathrm{MHz})\end{array}$ & Backend & Pulse phase bins & ToAs & Data span \\
\hline Nanshan $25 \mathrm{~m}$ & Room-temp. & 1540 & 320 & $\mathrm{AFB}$ & 256 & 110 & Jan 06, 2000 - Jul 25, 2002 \\
\hline Nanshan $25 \mathrm{~m}$ & Cryogenic & 1540 & 320 & DFB & 512 & 69 & Jan 18, 2010 - Mar 04, 2014 \\
\hline Parkes $64 \mathrm{~m}$ & Multibeam & 1374 & 288 & AFB & 512 & 20 & Dec 01, 2000 - Sep 30, 2006 \\
\hline Parkes $64 \mathrm{~m}$ & Multibeam & 1369 & 256 & PDFB3 & 1024 & 56 & Jul 24, 2008 - Feb 04, 2013 \\
\hline Parkes $64 \mathrm{~m}$ & Multibeam & 1369 & 256 & PDFB4 & 1024 & 8 & Feb 19, 2009 - Nov 10, 2009 \\
\hline Parkes $64 \mathrm{~m}$ & $1050 \mathrm{~cm}$ & 3094 & 1024 & PDFB2 & 1024 & 5 & Jul 23, 2007 - Jul 23, 2008 \\
\hline Parkes $64 \mathrm{~m}$ & $1050 \mathrm{~cm}$ & 3094 & 1024 & PDFB4 & 1024 & 9 & Jan 01, 2009 - Jan 18, 2013 \\
\hline
\end{tabular}

sic sources, such as superfluid turbulence (Greenstein 1970; Melatos \& Link 2014), microglitches (Cordes \& Downs 1985; Melatos et al. 2008), variable coupling between the crust and liquid interior or pinned and corotating regions (Alpar et al 1986; Jones 1990), fluctuations in the spin-down torque (Cheng 1987; Urama et al 2006), postglitch recovery (Johnston \& Galloway 1999), magnetospheric state switching (e.g., Lyne et al 2010), asteroid belts (Cordes \& Shannon 2008), evolution of the magnetic inclination angle (Yi \& Zhang 2015) and fluctuations in the magnetosphere (Ou et al. 2016).

PSR J1757-2421 (B1754-24) was discovered during a 1974 pulsar survey with Parkes 64m radio telescope (Komesaroff 1974). This star has a spin period $P=0.234 \mathrm{~s}$ and period derivative $\dot{P}=1.29 \times 10^{-14} \mathrm{~s} \mathrm{~s}^{-1}$. It has a characteristic age $\tau_{\mathrm{c}}=P /(2 \dot{P})=2.87 \times 10^{5}$ yr and a surface dipole magnetic field strength of $1.76 \times 10^{12} \mathrm{G}$. Hobbs et al. (2005) obtained a proper motion of PSR J1757-2421 in ecliptic longitude of $-17(5)$ mas $\mathrm{yr}^{-1}$ from pulse timing observations. Even though PSR J1757-2421 is energetic with a spin-down power $(\dot{E})$ of $4.0 \times 10^{34} \mathrm{erg} \mathrm{s}^{-1}$ and it is on the list of gammaray pulsar candidates for the Fermi Telescope (Smith et al. 2008), there is no report of detection of gamma-ray emission from this pulsar ${ }^{2}$.

In this article, we report on the pulsar profiles and timing behaviour for PSR J1757-2421, especially the large glitch which occurred in 2011.

\section{OBSERVATIONS}

In the pulsar timing program carried out with the Nanshan 25-m radio telescope located in Xinjiang, China, observations were taken with a receiver which has a bandwidth of $320 \mathrm{MHz}$ centred at $1540 \mathrm{MHz}$. The monitoring of PSR J1757-2421 was conducted with an observing cadence of three times per month and a 16-min duration. Early observations prior to 2010 were taken using an analogue filterbank (AFB) with $128 \times 2.5 \mathrm{MHz}$ subchannels and are described in Wang et al. (2001). After January 1, 2010, data were also obtained with a digital filterbank (DFB) which was configured to have 8-bit sampling and $1024 \times 0.5 \mathrm{MHz}$ channels which more than cover the $320 \mathrm{MHz}$ receiver bandwidth. The data are folded on-line with sub-integration times of 1

\footnotetext{
2 https://confluence.slac.stanford.edu/display/GLAMCOG/ Public+List+of+LAT-Detected+Gamma-Ray+Pulsars
}

min for the AFB and $30 \mathrm{~s}$ for the DFB, then written to disk with 256 bins across the pulse profile for the AFB and 512 bins for the DFB. The timing data presented were collected between January 6, 2000 and March 4, 2014 as detailed in Table 1.

Timing data from the Parkes 64-m radio telescope are also included, where the observations were carried out between December 2000 and February 2013 with a central observing frequency close to $1.37 \mathrm{GHz}$. Several observations of this pulsar were also made with the $10-\mathrm{cm}$ receiver which has a bandwidth of $1024 \mathrm{MHz}$ centred at $3094 \mathrm{MHz}$. An analogue filterbank and a series of digital filterbanks are used to acquire the raw data which are openly accessible via the CSIRO Data Access Portal ${ }^{3}$ (Hobbs et al. 2011).

The data were reduced using the PSRCHIVE pulsar analysis system (Hotan et al. 2004) to remove radio-frequency interference, and to dedisperse and average the multi-channel data to form mean pulse profiles. The times of arrival (ToAs) of pulses were derived by cross-correlation with templates or standard profiles which were obtained by summing all available data for each system. After that, the timing analysis was performed with the pulsar timing package TEMPO2, (Hobbs et al. 2006). The pulse ToAs at the observatories were referred to the solar system barycenter (SSB) employing the solar system ephemeris DE421 (Folkner et al. 2008). Then the ToAs at the SSB were fitted with the standard model of the pulse phase, $\phi(t)$, as a function of time, $t$ :

$\phi=\phi_{0}+\nu\left(t-t_{0}\right)+\frac{1}{2} \dot{\nu}\left(t-t_{0}\right)^{2}+\frac{1}{6} \ddot{\nu}\left(t-t_{0}\right)^{3}$

where $\phi_{0}$ is the phase at time $t_{0}$ and $\nu, \dot{\nu}$ and $\ddot{\nu}$ are the spin frequency, frequency derivative and frequency second derivative respectively. A glitch will result in an additional pulse phase modelled by the equation (Edwards et al. 2006):

$\phi_{\mathrm{g}}=\Delta \phi+\Delta \nu_{\mathrm{p}}\left(t-t_{\mathrm{g}}\right)+\frac{1}{2} \Delta \dot{\nu}_{\mathrm{p}}\left(t-t_{\mathrm{g}}\right)^{2}+\left[1-e^{-\left(t-t_{\mathrm{g}}\right) / \tau_{\mathrm{d}}}\right] \Delta \nu_{\mathrm{d}} \tau_{\mathrm{d}}$

where $t_{\mathrm{g}}$ is the glitch epoch and $\Delta \phi$ is an offset of pulse phase between the pre- and post-glitch data. The glitch event is characterised by permanent increments in the spin frequency $\Delta \nu_{\mathrm{p}}$ and first frequency derivative $\Delta \dot{\nu}_{\mathrm{p}}$ and a transient frequency increment $\Delta \nu_{\mathrm{d}}$ which decays exponentially with a time scale $\tau_{\mathrm{d}}$. Since ToA uncertainties are often underestimated because of instrumental and physical effects,

3 https://data.csiro.au/dap/ 
an additional term was added in quadrature to the ToA uncertainties. Quoted uncertainties are twice the formal standard deviation given by TEMPO2 (Tables 3 and 4). The offsets between Nanshan AFB data, Nanshan DFB data and Parkes data are estimated using TEMPO2. There are no obvious offsets between the different DFB systems at Parkes. Timing solutions are quoted in the TCB system referenced to TT(BIPM2013) ${ }^{4}$.

\section{ANALYSIS AND RESULTS}

\subsection{Pulse profiles}

Average pulse profiles are formed by adding the aligned preglitch data. The profiles at $1369 \mathrm{MHz}, 1540 \mathrm{MHz}$ and 3094 $\mathrm{MHz}$ are shown in Figure 1, where the pulse peaks are normalised to unity. We average the Nanshan DFB and Parkes profiles to 256 bins to match the Nanshan AFB data. At all three frequencies the trailing component is dominant, but the leading component is relatively much stronger at 3094 $\mathrm{MHz}$. At $1540 \mathrm{MHz}$, the width of pulse at $50 \%$ of peak (w50) is $11.02(14) \mathrm{ms}$, which implies a duty cycle of $\sim 4.71(6) \%$. It is useful to fit the observed profiles by Gaussian components using

$I(\phi)=\sum_{i=1}^{M} I_{\mathrm{i}} e^{-4 \ln 2\left(\phi-\phi_{\mathrm{i}}\right)^{2} / w_{\mathrm{i}}^{2}}$

where $I_{\mathrm{i}}$ is the amplitude of the $i$ th Gaussian component, $\phi_{\mathrm{i}}$ is the peak phase, and $w_{\mathrm{i}}$ is the full width at half peak. These parameters were determined using the LevenbergMarquardt algorithm (Press et al. 1992). Three components were fitted and the fitting results are presented in Table 2 and Figure 2. It is shown clearly in Figure 3 that the phase separations of the two outermost components $\left(\phi_{3}-\phi_{1}\right)$ increases with increasing frequency. However, the summed width of the Gaussian components $\left(w_{1}+w_{2}+w_{3}\right)$ is smaller at $3.1 \mathrm{GHz}$.

4 http://www. bipm.org

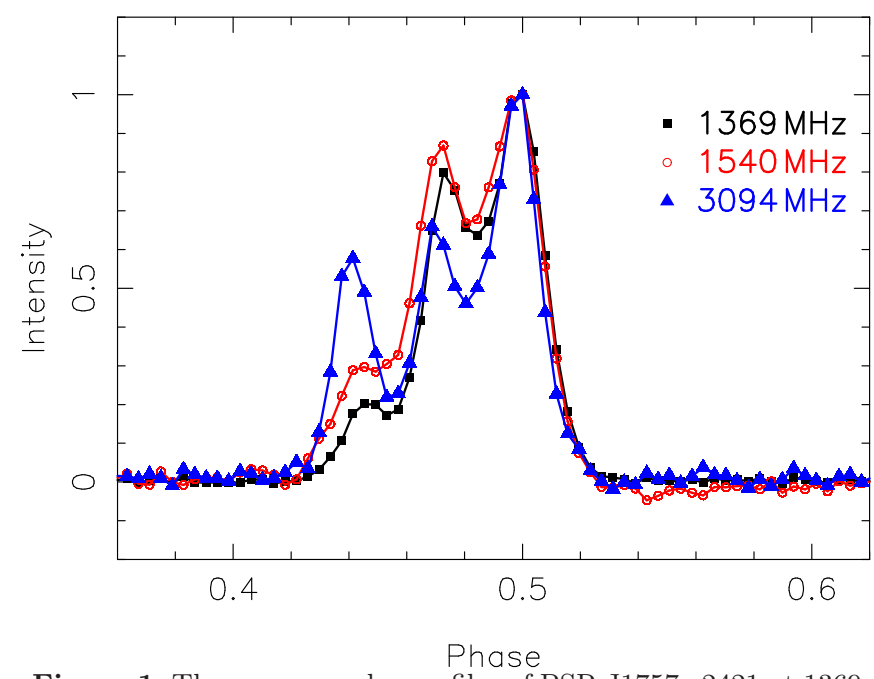

Figure 1. The average pulse profiles of PSR J1757-2421 at 1369 $\mathrm{MHz}$ (black), $1540 \mathrm{MHz}$ (red) and $3094 \mathrm{MHz}$ (blue). The pulse peaks are normalised to unity.
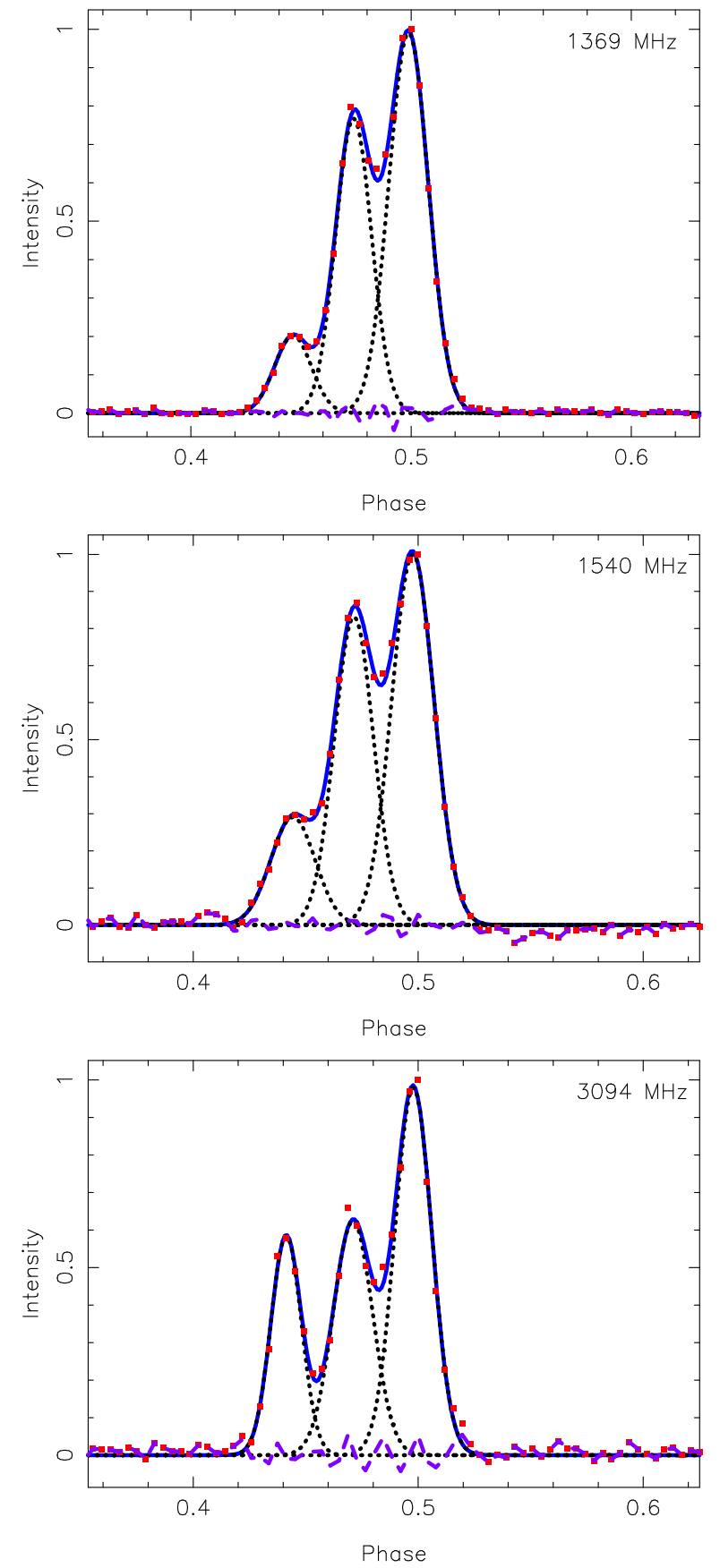

Figure 2. Observed pulse profiles of PSR J1757-2421 with fitted Gaussian components. Residuals from the Gaussian fit are shown as a dashed line in each plot.

For most pulsars, the radio spectrum at frequencies above about $1 \mathrm{GHz}$ obeys a power law $S \sim \nu_{r}^{\alpha}$, where $\nu_{r}$ is the radio frequency and the observed spectral index $\alpha$ is generally between -1.5 and -1.8 . The relative phase-resolved spectral indices shown in Figure 4 are determined using the normalized profiles at $1369 \mathrm{MHz}, 1540 \mathrm{MHz}$ and $3094 \mathrm{MHz}$ (Figure 1). The on-pulse region is taken to be where the signal exceeds three times the baseline rms noise for all frequencies. As the Nanshan observations were not calibrated, we determine absolute spectral indices using the Parkes 1369 and $3094 \mathrm{MHz}$ data, flux-calibrated using associated obser- 
Table 2. Parameters of the fitted Gaussian profile components for PSR J1757-2421, $I_{\mathrm{i}}$ is the amplitude of the $i$ th Gaussian component, $\phi_{\mathrm{i}}$ is the peak phase, and $w_{\mathrm{i}}$ is the full width at half peak.

\begin{tabular}{llllllllll}
\hline Frequency & $I_{1}$ & $\phi_{1}$ & $w_{1}$ & $I_{2}$ & $\phi_{2}$ & $w_{2}$ & $I_{3}$ & $\phi_{3}$ & $w_{3}$ \\
\hline $1369 \mathrm{MHz}$ & $0.2019(3)$ & $0.4463(2)$ & $0.0196(3)$ & $0.7880(3)$ & $0.4739(1)$ & $0.0193(3)$ & $0.9902(3)$ & $0.49885(6)$ & $0.0212(3)$ \\
$1540 \mathrm{MHz}$ & $0.2913(9)$ & $0.4443(7)$ & $0.0232(10)$ & $0.8320(11)$ & $0.4714(4)$ & $0.0205(11)$ & $0.9998(10)$ & $0.4976(2)$ & $0.0222(10)$ \\
$3094 \mathrm{MHz}$ & $0.5847(11)$ & $0.4413(2)$ & $0.0160(11)$ & $0.6236(10)$ & $0.4710(3)$ & $0.0208(10)$ & $0.9795(10)$ & $0.4978(2)$ & $0.0193(10)$ \\
\hline
\end{tabular}
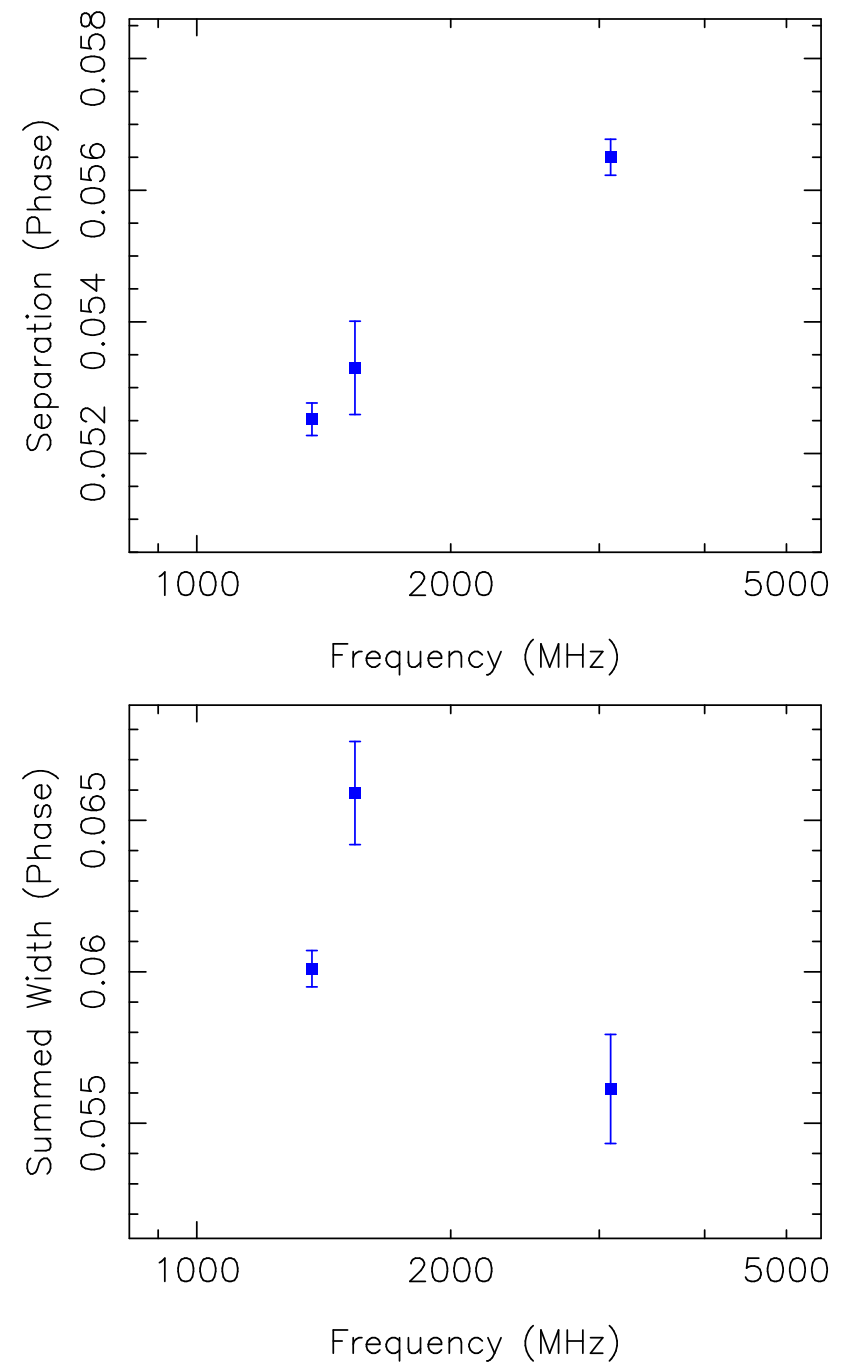

Figure 3. Top: The phase separation of the two outermost components presented in Figure 2 . Bottom: The summed width of the three Gaussian components shown in Figure 2.

vations of Hydra A. Figure 4 shows that the spectral index for the leading component (phase $0.43-0.45$ ) is close to zero, whereas the central and trailing components have a much steeper spectrum with $\alpha \sim-4$.0. The spectral index has its minimum value between the central and trailing components.

\subsection{The glitch in PSR J1757-2421}

We adopt the astrometric parameters and dispersion measure obtained by Hobbs et al. (2005) with the position and proper motion fixed in this analysis. It is not possible to ob-

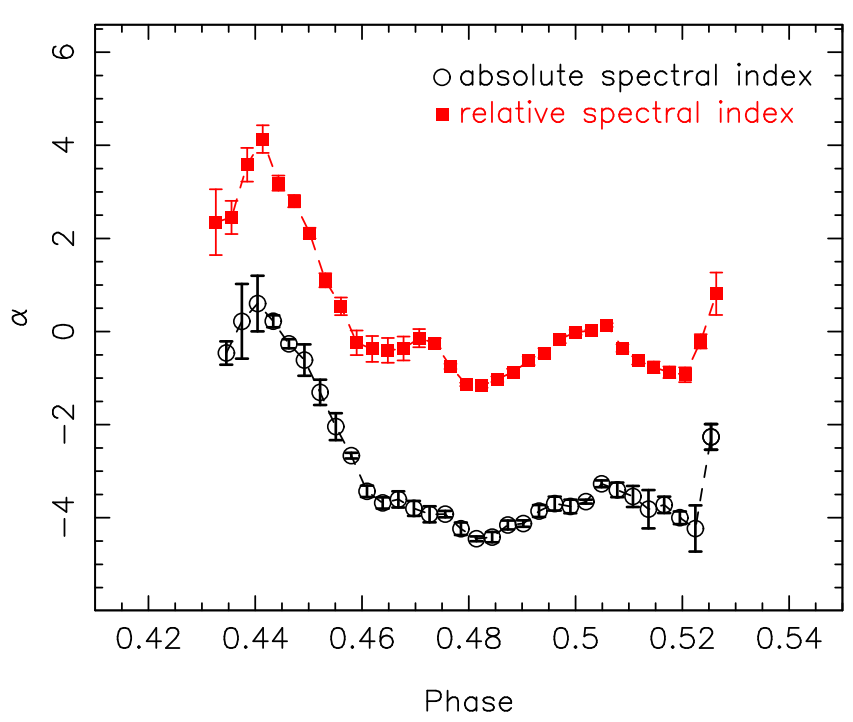

Figure 4. The phase resolved spectral index for PSR J1757-2421.

tain a phase-connected solution for PSR J1757-2421 with the whole data set, as a previously unreported very large glitch occurred in May 2011. The rotational parameters for PSR J1757-2421 from fitting of the timing model (Equation $1)$ to the pre- and post-glitch data are given in Table 3 . To examine the spin behaviour of PSR J1757-2421, the spin frequency $\nu$ and frequency derivative $\dot{\nu}$ were derived from independent fits to short sections of data, each of which typically span $\sim 60 \mathrm{~d}$. Figure 5 shows an overview of the spindown of PSR J1757-2421, indicating that a large glitch with $\Delta \nu \sim 33 \times 10^{-6} \mathrm{~Hz}$ occurred between MJD 55697 and 55708 (May 16, 2011 and May 27, 2011). Assuming a glitch epoch of MJD 55702 and using the data between MJD 54515 and 55795 , the spin parameters are extrapolated to the glitch epoch with the pre- and post-glitch solutions given in Table 3. It is noted that here we don't take the exponential decays into account. Then, the glitch parameters are estimated by calculating the fractional jump in $\nu$ and $\dot{\nu}$, which results in $\Delta \nu_{\mathrm{g}} / \nu \sim 7809(2) \times 10^{-9}$ and $\Delta \dot{\nu}_{\mathrm{g}} / \dot{\nu} \sim 67(16) \times 10^{-3}$. The frequent timing observations of this pulsar make it possible to track the recovery process relatively thoroughly.

For this pulsar, the residual frequencies and frequencyderivatives have been obtained from the values at the various epochs by subtracting the pre-glitch models. Figure $5(\mathrm{~b})$ is obtained by the subtraction of the mean frequency values on each side of the glitch epoch. Although most of the frequency jump persists beyond the end of the data span, the expanded plot shown in Figure 5(b) and the plot of $\dot{\nu}$ presented in Figure 5(c) show that there is an initial exponential decay.

Because of the large amplitude of the glitch, the final re- 
Table 3. Timing parameters for PSR J1757-2421. The uncertainties for the measured quantities $(\nu, \dot{\nu}, \ddot{\nu})$ are twice the formal standard deviations given by TEMPO2

\begin{tabular}{|c|c|c|}
\hline Parameters & Pre-glitch & Post-glitch \\
\hline Pulsar name............................. & J1757-2421 & J1757-2421 \\
\hline MJD range $\ldots \ldots \ldots \ldots \ldots \ldots \ldots \ldots \ldots \ldots \ldots \ldots \ldots \ldots$ & $51549-55697$ & $55707.9-56719.1$ \\
\hline 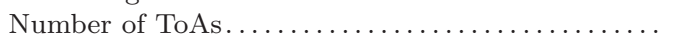 & 407 & 84 \\
\hline Rms timing residual $(\mu s) \ldots \ldots \ldots \ldots \ldots \ldots$ & 3203 & 7739 \\
\hline Weighted fit $\ldots \ldots \ldots \ldots \ldots \ldots \ldots \ldots \ldots$ & $\mathrm{Y}$ & $\mathrm{Y}$ \\
\hline Reduced $\chi^{2}$ value $\ldots \ldots \ldots \ldots \ldots \ldots \ldots \ldots$ & 376.3 & $24454^{\dagger}$ \\
\hline \multicolumn{3}{|c|}{ Measured Quantities } \\
\hline Pulse frequency, $\nu\left(\mathrm{s}^{-1}\right) \ldots \ldots \ldots \ldots \ldots \ldots \ldots$ & $4.27159264655(4)$ & $4.2715730980(8)$ \\
\hline First derivative of pulse frequency, $\dot{\nu}\left(\mathrm{s}^{-2}\right) \ldots \ldots \ldots$ & $-2.356777(3) \times 10^{-13}$ & $-2.3729(3) \times 10^{-13}$ \\
\hline Second derivative of pulse frequency, $\ddot{\nu}\left(\mathrm{s}^{-3}\right) \ldots$ & $-4.80(13) \times 10^{-25}$ & $3.4(4) \times 10^{-23}$ \\
\hline \multicolumn{3}{|c|}{ Set Quantities } \\
\hline Ecliptic longitude (deg.) ..................... & $269.428041(5)$ & $269.428041(5)$ \\
\hline Ecliptic latitude (deg.) $\ldots \ldots \ldots \ldots \ldots \ldots \ldots \ldots$ & $-0.9307(4)$ & $-0.9307(4)$ \\
\hline Epoch of frequency determination (MJD) ........ & 53623 & 56213 \\
\hline Epoch of position determination (MJD) ......... & 53623 & 53623 \\
\hline Epoch of dispersion measure determination (MJD) & 49909 & 49909 \\
\hline Dispersion measure, $\mathrm{DM}\left(\mathrm{cm}^{-3} \mathrm{pc}\right) \ldots \ldots \ldots \ldots$ & 179.454 & $179.454^{\ddagger}$ \\
\hline Proper motion in ecliptic longitude $\left(\right.$ mas $\left.\mathrm{yr}^{-1}\right)$ & $-17(5)$ & $-17(5)$ \\
\hline \multicolumn{3}{|c|}{ Assumptions } \\
\hline Reference time scale $\ldots \ldots \ldots \ldots \ldots \ldots \ldots \ldots$ & TT(BIPM2013) & TT(BIPM2013) \\
\hline Solar system ephemeris model $\ldots \ldots \ldots \ldots \ldots \ldots$ & DE421 & DE421 \\
\hline Time units $\ldots \ldots \ldots \ldots \ldots \ldots \ldots \ldots \ldots \ldots \ldots$ & TCB & TCB \\
\hline
\end{tabular}

${ }^{\dagger}$ The reduced $\chi^{2}$ for post-glitch is large because of the unmodelled glitch.

¥ The reported variation of DM (Hobbs et al. 2004) is not considered in this article.

sult was obtained in several steps. For the first step, fits are to a data span that covers $780 \mathrm{~d}$ from MJD 55213 to MJD 55993. A single decay with an assumed time scale of 100 days is fitted to the ToAs, resulting values of $\Delta \nu_{\mathrm{p}} \sim 3.325 \times 10^{-5}$ Hz. Post-fit residuals from the fit are shown in the upper panel of Figure 6. It is obvious from this plot that there is an additional initial decay with time constant $\sim 15$ days. Fitting for the two decay terms gives $\Delta \nu_{\mathrm{d} 1} \sim 4.5(23) \times 10^{-8} \mathrm{~Hz}$ and $\tau_{\mathrm{d} 1}=15(6) \mathrm{d}, \Delta \nu_{\mathrm{d} 2} \sim 7.3(8) \times 10^{-8} \mathrm{~Hz}$ and $\tau_{\mathrm{d} 2}=97(15)$ d. Residuals for this fit shown in the lower panel of Figure 6 demonstrate that the observed ToAs over this data span are very well modeled by increments in $\nu$ and $\dot{\nu}$ together with two exponential decays with time constants around 15 and 100 days, respectively. The resulting glitch parameters are given in Table 4 . These values are a little larger than those obtained with the extrapolation method which doesn't take the exponential recovery into account. Figure 5(a) shows that the permanent jump in the frequency is dominant. The fitted $\Delta \nu_{\mathrm{p}}=33.263(6) \times 10^{-6} \mathrm{~Hz}$, which implies that $99.65 \%$ of the initial frequency jump does not decay. For this event, the degree of recovery $\left(Q=\Delta \nu_{\mathrm{d}} / \Delta \nu_{\mathrm{g}}\right)$, where $\Delta \nu_{\mathrm{g}}=\Delta \nu_{\mathrm{p}}+\Delta \nu_{\mathrm{d} 1}+\Delta \nu_{\mathrm{d} 2}$, is very small with $Q \sim 0.35(9) \%$. This is consistent with earlier results that show small values of $Q$, typically around 0.01 , are commonly detected in large glitches (Yu et al. 2013).

The activity parameter for glitching pulsars is defined as $A_{\mathrm{g}}=\frac{1}{T} \sum \frac{\Delta \nu_{g}}{\nu}$, where $T$ is the total data span (McKenna \& Lyne 1990). A value of $A_{\mathrm{g}} \sim 0.55 \times 10^{-9} \mathrm{~d}^{-1}$ is obtained for PSR J1757-2421, assuming that it has glitched once since it was discovered in 1974 and that the 39 years
Table 4. Glitch parameters for PSR J1757-2421. The uncertainties for the spin parameters are twice the values produced by TEMPO2

\begin{tabular}{ll}
\hline Glitch epoch (MJD) & $55702(6)$ \\
$\Delta \nu_{\mathrm{p}}(\mathrm{Hz})$ & $33.263(6) \times 10^{-6}$ \\
$\Delta \dot{\nu}_{\mathrm{p}}\left(\mathrm{s}^{-2}\right)$ & $-1.8(4) \times 10^{-15}$ \\
$\Delta \nu_{\mathrm{d} 1}(\mathrm{~Hz})$ & $4.5(23) \times 10^{-8}$ \\
$\tau_{\mathrm{d} 1}(\mathrm{~d})$ & $15(6)$ \\
$\Delta \nu_{\mathrm{d} 2}(\mathrm{~Hz})$ & $7.3(8) \times 10^{-8}$ \\
$\tau_{\mathrm{d} 2}(\mathrm{~d})$ & $97(15)$ \\
$\Delta \nu_{\mathrm{g}}(\mathrm{Hz})$ & $33.381(14) \times 10^{-6}$ \\
$\Delta \nu_{\mathrm{g}} / \nu$ & $7815(3) \times 10^{-9}$ \\
$\Delta \dot{\nu}_{\mathrm{g}} / \dot{\nu}$ & $196(56) \times 10^{-3}$ \\
$Q\left(=\Delta \nu_{\mathrm{d}} / \Delta \nu_{\mathrm{g}}\right)$ & $0.35(9) \%$ \\
\hline
\end{tabular}

since then is the average glitch interval. Figure 5(c) shows that there is also a permanent jump in frequency derivative $(\dot{\nu})$ of $\dot{\nu}_{\mathrm{p}} \sim-1.8(4) \times 10^{-15} \mathrm{~s}^{-2}$. This is small with the fractional increase $\Delta \dot{\nu}_{\mathrm{p}} / \dot{\nu} \sim 0.76(9) \%$, less than the value of $1.7 \%$ found by Lyne et al. (2000) to represent most glitches. The total increment in $\dot{\nu}$ is given by

$\Delta \dot{\nu}_{\mathrm{g}}=\Delta \dot{\nu}_{\mathrm{p}}-\Delta \nu_{\mathrm{d} 1} / \tau_{\mathrm{d} 1}-\Delta \nu_{\mathrm{d} 2} / \tau_{\mathrm{d} 2}$

This gives $\Delta \dot{\nu}_{\mathrm{g}} / \dot{\nu}=0.196(56)$, much larger than the permanent increment, but with large uncertainty because of the uncertainties in the decay parameters. 


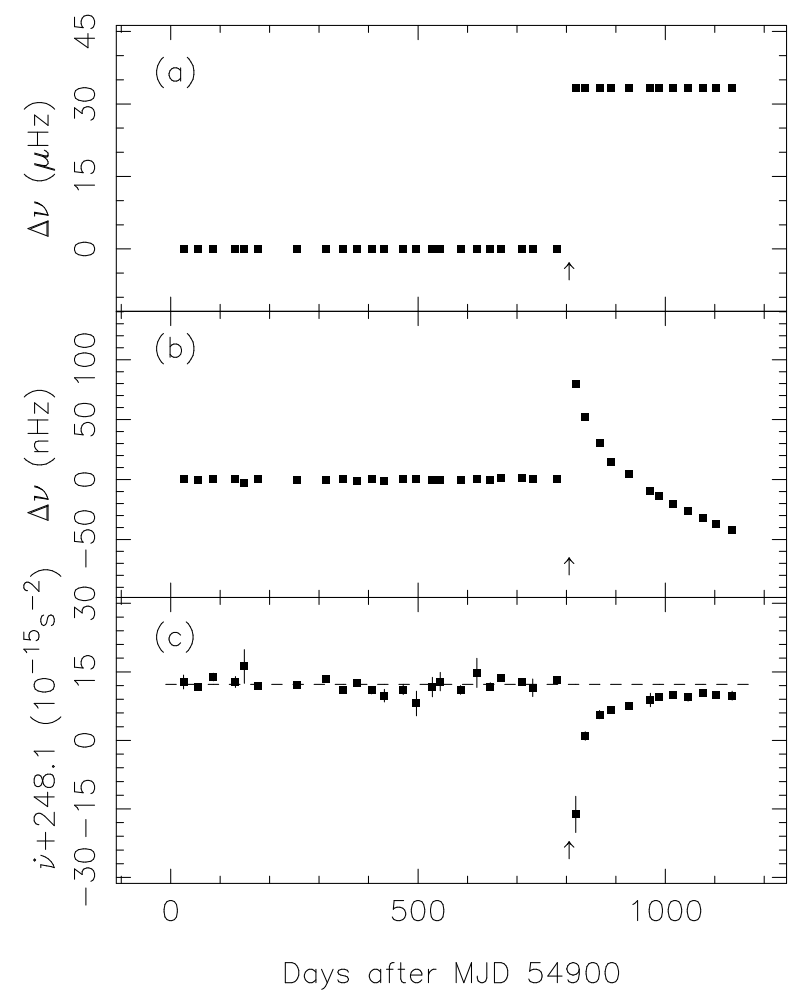

Figure 5. The glitch in PSR J1757-2421. The top panel shows the spin-frequency residuals $\Delta \nu$ relative to the pre-glitch spindown solution; the middle panel is an expanded plot of $\Delta \nu$ where the mean post-glitch value is subtracted from the post-glitch data; the bottom panel shows the variations of $\dot{\nu}$, and the horizontal dashes indicate the average pre-glitch level. The glitch epoch is indicated by an arrow.

\subsection{Timing noise}

Figure 7 presents the pre-glitch timing residuals relative to a solution containing the spin frequency $\nu$, first derivative $\dot{\nu}$ and second derivative $\ddot{\nu}$ for PSR J1757-2421. It is obvious that there is significant red timing noise. The parameter $\Delta_{8}$ was introduced by Arzoumanian et al. (1994) to quantify the magnitude of timing noise. Its value is calculated as

$\Delta_{8}=\log \left(\frac{|\ddot{\nu}|}{6 \nu} t^{3}\right)$

where the spin-frequency, $\nu$, and its second derivative, $\ddot{\nu}$, are measured over interval of $t=10^{8} \mathrm{~s}$ (about $3 \mathrm{yr}$ ). As the pre-glitch data set for PSR J1757-2421 spans over 11 yr, we obtained four values by fitting over four 3 -yr segments, obtaining $\Delta_{8}$ ranging from $-2.33(1)$ to $-1.27(3)$. It was known that the $\Delta_{8}$ parameter is strongly correlated with the spin-down rate, with $\Delta_{8}=5.1+0.5 \log \dot{P}$ being obtained by Hobbs et al. (2010). For PSR J1757-2421 with $\dot{P}=12.9 \times 10^{-15} \mathrm{~s} \mathrm{~s}^{-1}$, this relation has a value of -1.84 , consistent with the measured values.

Coles et al. (2011) presented a method for analysing correlated timing noise. This procedure estimates the covariance matrix of timing residuals using generalized leastsquares fitting. The covariance matrix is used to perform a linear transformation based on the Cholesky decomposition of the covariance matrix that whitens both the residuals and timing model. Spectral leakage in the presence of strong red

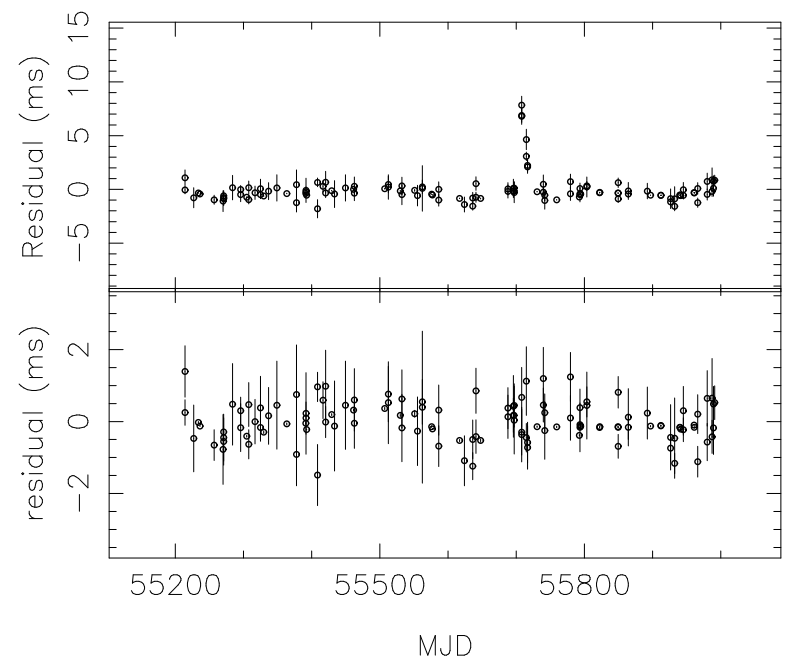

Figure 6. The timing residuals for PSR J1757-2421 between MJD 55214 and 55992. For the upper plot, one decay term is fitted, whereas two exponential decay terms are fitted for the lower plot.

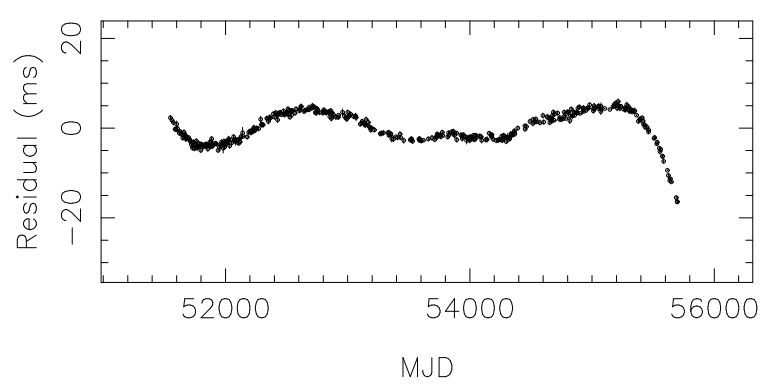

Figure 7. The timing residual of PSR J1757-2421 between MJD 51549 - 55696, with respect to the pre-glitch timing model in Table 3.

timing noise is minimized with pre-whitening using a difference filter. The low-frequency noise can be fitted with a power-law model $P(f)=A /\left[1+\left(f / f_{c}\right)^{2}\right]^{\alpha / 2}$, where $f$ is the modulation frequency, $A$ is the amplitude, $\alpha$ is the spectral exponent, and $f_{c}$ is the corner frequency. Through an iterative process, updated estimates of the spectrum can be achieved by using Lomb-Scargle periodogram after whitening the data using the Cholesky decomposition of the covariance matrix. Figure 8 presents the spectrum of the pre-glitch timing noise in PSR J1757-2421. The low frequency noise is well modeled by a power-law with corner frequency of $0.18 \mathrm{yr}^{-1}$ and spectral exponent of $\alpha \sim-5.9$, which is close to -6 . For the post-glitch data, in order to avoid the impact of the post-glitch recovery, we analyse the spectrum of the residual between MJD 55822 (100 days after the glitch epoch) and 56720. Figure 8 shows the spectrum of the postglitch timing noise, which is modeled on a power-law with spectral exponent of $\alpha \sim-6.2$ and a corner frequency of $0.4 \mathrm{yr}^{-1}$. Although, the corner frequency and spectral exponents are to some degree empirical, these indices suggest that both the pre-glitch and post-glitch noise can be modelled as a random walk in the spin-down $\dot{\nu}$. 
Table 5. Parameters for the glitches with two decay terms.

\begin{tabular}{lllllllll}
\hline PSR & $\begin{array}{l}\text { Epoch } \\
\text { (MJD) }\end{array}$ & $\begin{array}{l}\Delta \nu_{d 1} \\
\left(10^{-6} \mathrm{~Hz}\right)\end{array}$ & $Q_{1}$ & $\begin{array}{l}\tau_{d 1} \\
(\mathrm{~d})\end{array}$ & $\begin{array}{l}\Delta \nu_{d 2} \\
\left(10^{-6} \mathrm{~Hz}\right)\end{array}$ & $Q_{2}$ & $\begin{array}{c}\tau_{d 2} \\
(\mathrm{~d})\end{array}$ & Ref \\
\hline J0835-4510 & $40280(4)$ & $0.0518(5)$ & $0.00198(2)$ & $10(1)$ & $0.4665(13)$ & $0.01782(5)$ & $120(6)$ & Cordes et al (1988) \\
& $41192(8)$ & $0.0362(5)$ & $0.00158(2)$ & $4(1)$ & $0.300(2)$ & $0.01311(9$ & $94(5)$ & Cordes et al (1988) \\
& $42683(3)$ & $0.00968(11)$ & $0.000435(5)$ & $4.0(4)$ & $0.0786(4)$ & $0.003534(16)$ & $35(2)$ & Cordes et al (1988) \\
& $43693(12)$ & $0.0830(7)$ & $0.00242(2)$ & $6.0(6)$ & $0.3888(7)$ & $0.01134(2)$ & $75(3)$ & Cordes et al (1988) \\
& $44888.4(4)$ & $0.01036(10)$ & $0.000813(8)$ & $6.0(6)$ & $0.0242(5)$ & $0.00190(4)$ & $14(2)$ & Cordes et al (1988) \\
& $45192.1(5)$ & $0.05701(16)$ & $0.002483(7)$ & $3.0(6)$ & $0.1263(18)$ & $0.00550(8)$ & $21.5(2.0)$ & Cordes et al (1988) \\
& $46259(2)$ & $0.066(9)$ & $0.0037(5)$ & $6.5(5)$ & $2.76(1)$ & $0.1541(6)$ & $332(10)$ & McCulloch et al. (1987) \\
J1119-6127 & $47519.80360(8)$ & $0.1086(2)$ & $0.005385(10)$ & $4.62(2)$ & $3.396(8)$ & $0.1684(4)$ & $351(1)$ & McCulloch et al. (1990) \\
J1757-2421 & $54244(24)$ & $18.6(218)$ & $0.81(81)$ & $15.7(3)$ & $4.9(43)$ & $0.214(136)$ & $186(3)$ & Yu et al. (2013) \\
J1803-2137 & $55702(6)$ & $0.045(13)$ & $0.0013(8)$ & $15(6)$ & $0.073(4)$ & $0.0022(2)$ & $97(15)$ & this work \\
J2337+6151 & $53615(15)$ & $0.23(16)$ & $0.0094(65)$ & $12(2)$ & $0.080(15)$ & $0.00330(64)$ & $69(3)$ & Yu et al. (2013) \\
& & $0.19(3)$ & $0.0046(7)$ & $21.4(5)$ & $0.119(4)$ & $0.0029(1)$ & $147(2)$ & Yuan et al. (2010b) \\
\hline
\end{tabular}

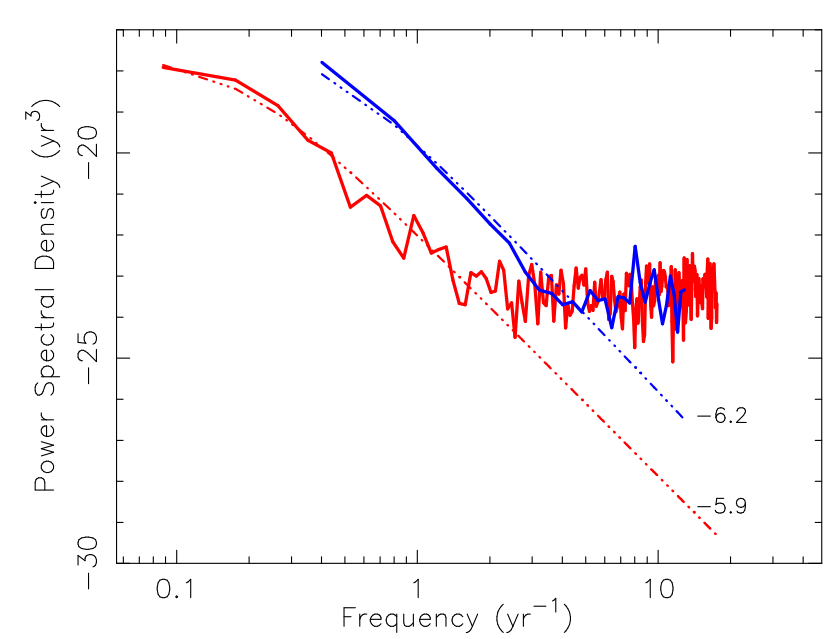

Figure 8. The observed power spectra of the pre-glitch timing noise (red) and post-glitch timing noise (blue) for PSR J1757-2421. The fitted noise models are shown as dashed lines with the spectral exponents presented.

\section{DISCUSSION}

The observations presented here show that the pulse profile exhibits strong frequency evolution. In numerous early investigations, it was found that the pulse component separation often decreases with increasing frequency (e.g. Thorsett 1991). This is usually interpreted in the context of the "magnetic-pole" model (Radhakrishnan \& Cooke 1969) as "radius-to-frequency" mapping (e.g., Cordes 1978) with the lower frequencies being emitted at greater altitudes above the magnetic pole. However, Chen \& Wang (2014) found that quite a few pulsars (19\% of 150 normal pulsars) showed wider profiles at high frequencies. Figure 3 shows that PSR J1757-2421 is clearly a member of this minority group.

The average pulse profile of PSR J1757-2421 is characterised by three components whose relative strengths vary with frequency with the leading component having a much flatter spectral index. At high frequencies the conal component, generated from the outer parts of the open field-line bundle, tends to dominate the profile (e.g., Lyne \& Manchester 1988). Such kind of profile evolution may lead to partial cones at certain frequencies or the broad- ening of the inner cone width at high frequency in the inverse Compton scattering model (Qiao \& Lin 1988). Wang et al. (2014) account for it as an asymmetrical spectral distribution across the flux tubes in the pulsar magnetosphere.

This is the first detection of a glitch in the energetic pulsar PSR J1757-2421 since it was discovered in 1973. This is a Vela-like event with a relative amplitude of $\Delta \nu_{\mathrm{g}} / \nu \sim$ $7.8 \times 10^{-6}$, identifying it as large glitch. Such a large event has not been previously detected in pulsars with characteristic age $\tau_{c} \sim 3 \times 10^{5} \mathrm{yr}$. Most of the large glitches are detected in pulsars with characteristic ages smaller than $10^{5}$ yr. For those radio pulsars with characteristic ages in the range $2.3 \times 10^{6}$ to $3.2 \times 10^{6} \mathrm{yr}$, similar to PSR J1757-2421, only two (PSRs J1743-3150 and J1825-0935) have glitched, and these were small glitches (Espinoza et al. 2011). However, large glitches have been detected in some pulsars older than PSR J1757-2421, such as J0358+5413 (Espinoza et al. 2011).

For the glitch in PSR J1757-2421, the permanent jump in the spin frequency is dominant, with $Q \sim 0.35 \%$, which is consistent with the small $Q$ usually seen in large glitches. PSR J1757-2421 shows an exponential recovery following the glitch with two identifiable decay terms having time constants of approximately $15 \mathrm{~d}$ and $97 \mathrm{~d}$ respectively. Including PSR J1757-2421, five pulsars have shown evidence of two exponential decay terms in one event as listed in Table 5 . where the shorter term ranges from $3 \mathrm{~d}$ to $22 \mathrm{~d}$, and the longer range from $14 \mathrm{~d}$ to $332 \mathrm{~d}$. Because observational gaps are often several weeks, there could be more fast decays (with time scales less than $20 \mathrm{~d}$ ) which have been missed. In this case, the magnitude of the jumps in $\nu$ and $\dot{\nu}$ could be much larger.

The theoretical understanding of pulsar glitches includes trigger mechanisms which seek to explain how the glitch originates and to predict glitch sizes, waiting times and their distributions, and relaxation mechanisms which explain the behavior and timescales of the post-glitch recovery. For large glitches, a popular model is a vortex unpinning process in the superfluid components of the star (Anderson \& Itoh 1975; Alpar et al. 1984). The observed post-glitch exponential recoveries have been explained as the re-establishment of an equilibrium between pinning and un- 
pinning in a vortex-creep region interior to a neutron star (Alpar et al. 1993; Lyne et al. 2000).

Link et al. (1999) have defined a coupling parameter, $G=2 \tau_{\mathrm{c}} A_{\mathrm{g}}$, which equals the minimum fraction of the moment of inertia that transfers angular momentum to the crust in glitches. Since $A_{\mathrm{g}}$ for PSR J1757-2421 is $\sim 0.55 \times$ $10^{-9} \mathrm{~d}^{-1}$, the corresponding $G$ is $\sim 11 \%$. Chamel (2005, 2012) found that the effect of entrainment makes it very difficult to move superfluid neutrons relative to the crust lattice. As a result, Andersson et al. (2012) and Chamel (2013) found that the previous calculations of Link et al. (1999) underestimate by a factor of about four the moment of inertia required by observed glitches and that the superfluid reservoir in the crust of neutron stars is insufficient to produce the observed size and frequency of glitches. For PSR J1757-2421, it requires a reservoir comprising $\sim 45 \%$ of the total moment of inertia. Delsate et al. (2016) calculated the crustal moment of inertia of glitching pulsars by employing a series of different unified dense-matter equations of state and found values less than $20 \%$. Although the computed $G$ for PSR J1757-2421 may be an over-estimate since only one glitch has been detected, this result suggests that transfer of angular momentum from the core superfluid may be required to account for the large glitch in this relatively old pulsar.

\section{ACKNOWLEDGEMENTS}

This work is supported by National Basic Research Program of China (2015CB857100), West Light Foundation of CAS (No. ZD201302), National Natural Science Foundation of China (NSFC No. 11173041) and Strategic Priority Research Programme (B) of Chinese Academy of Sciences (No. XDB23000000 and XDB09000000). JPY acknowledge the FAST Fellowship which is supported by Special Funding for Advanced Users, budgeted and administrated by CAMS. JBW acknowledges support from NSFC Grant (11403086, U1431107) and West light Foundation of CAS (XBBS201322). XZ is supported by NSFC (11373006, 11003034).

We thank the referee for helpful comments, and H. G. Wang, J. L. Chen, K. J. Lee and H. Tong for useful discussions. This work is based on observations made with the Urumqi Nanshan $25 \mathrm{~m}$ Telescope, which is operated by $\mathrm{XAO}$ and the Key Laboratory of Radio Astronomy, Chinese Academy of Sciences. The Parkes radio telescope is part of the Australia Telescope, which is funded by the Commonwealth of Australia for operation as a National Facility managed by the Commonwealth Scientific and Industrial Research Organisation.

\section{REFERENCES}

Alpar M. A., Anderson P. W., Pines D., Shaham J., 1981, ApJ, $249,29 \mathrm{~L}$

Alpar M. A., Pines D., Anderson P. W., Shaham J., 1984, ApJ, 276,325

Alpar M. A., Chau H. F., Cheng K. S., Pines, David, 1993, ApJ. 409,345

Alpar M. A., Nandkumar R., Pines D., 1986, ApJ, 311, 197
Andersson N., Glampedakis K., Ho W. C. G., Espinoza C. M., 2012, PhRvL, 109, 241103

Anderson P. W., Itoh N., 1975, Nature, 256, 25

Arzoumanian Z., Nice D. J., Taylor J. H., Thorsett S. E., 1994, ApJ, 422, 671

Baym G., Pines D., 1971, Ann. Phys., 66, 816

Boynton P. E., Groth E. J., Hutchinson D. P., Nanos G. P., Partridge R. B., Wilkinson D. T., 1972, ApJ, 175, 217-241

Chamel N., 2005, Nucl. Phys. A 747, 109128

Chamel N., 2012, Phys. Rev. C 85, 035801

Chamel N., 2013, PhRvL, 110a, 1101

Chen J. L., Wang H. G., 2014, ApJS, 215, 11

Cheng K. S., 1987, ApJ, 321, 799

Coles W., Hobbs G., Champion D. J., Manchester R. N., Verbiest J. P. W., 2011, MNRAS, 418, 561

Cordes J. M., 1978, ApJ, 222, 1006

Cordes J. M., Downs G. S., 1985, ApJS, 59, 343-382

Cordes J. M., Downs G. S., Krause-Polstorff J., 1988, ApJ, 330, 847

Cordes J. M. \& Shannon R. M., 2008, ApJ, 682, 1152

Delsate T., Chamel N., Gürlebeck N., Fantina A. F., Pearson J. M., Ducoin C., 2016, PhRvD, 94, 023008

Edwards R. T., Hobbs G. B., Manchester R. N., 2006, MNRAS, 372,1549

Espinoza C. M., Lyne A. G., Stappers B. W., Kramer M., 2011, MNRAS, 414, 1679

Folkner W. M., Williams J. G., Boggs D. H., 2008, JPL IOM 343R-08-00

Glampedakis K., Andersson N., 2009, PhRvL, 102, 1101,

Greenstein G., 1970, Nature, 227, 791-794

Haskell B., Antonopoulou D., 2014, MNRAS, 438, 16

Hobbs G., Lyne A. G., Kramer M., Martin C. E., Jordan C., 2004, MNRAS, 353, 1311

Hobbs G., Lorimer D. R., Lyne A. G., Kramer M., 2005, MNRAS, 360,974

Hobbs G. B., Edwards R. T., Manchester R. N., 2006, MNRAS, 369,655

Hobbs G., Lyne A. G., Kramer M., 2010, MNRAS, 402, 10271048 ,

Hobbs G., et al., 2011, PASA, 28, 202

Hotan A. W., van Straten W., Manchester R. N., 2004, PASA, 21,302

Jones P. B., 1990, MNRAS, 246, 364

Johnston S., Galloway D., 1999, MNRAS, 306, 50

Komesaroff M. M., 1974, Unpublished

Link B., Epstein R. I., Lattimer J. M., 1999, Phys. Rev. Lett. 83, 3362-3365.

Lyne A., Hobbs G., Kramer M., Stairs I., Stappers B, 2010, Science, 329, 408

Lyne A. G., Manchester R. N., 1988, MNRAS, 234, 477

Lyne A. G., Shemar S. L., Graham-Smith F., 2000, MNRAS, 315, 534

Manchester R. N., Hobbs G. B., Teoh A., Hobbs M., 2005, MNRAS, 129, 1993

McKenna J., Lyne A. G., 1990, Nature, 343, 349

McCulloch P. M., Klekociuk A. R., Hamilton P. A, Royle G. W. R., 1987, AuJPh, 40, 725

McCulloch P. M., Hamilton P. A, McConnell D., King E. A., 1990, Nature, 346, 30

Melatos A., Link B., 2014, MNRAS, 437, 21-31

Melatos A., Peralta C., Wyithe J. S. B., 2008, ApJ, 672, 1103

Ou Z. W., Tong H., Kou F. F., Ding G. Q., 2016, MNRAS, 457, 39220

Press W. H., Teukolsky S. A., Vetterling V. T., Flannery B. P., 1992, Numerical Recipes in C, Cambridge University Press, Cambridge, England

Qiao G. J., Lin W. P., 1998, AA, 333, 172

Radhakrishnan V., Cooke D. J., 1969, ApJ, 3, 225 
Radhakrishnan V., Manchester R. N., 1969, Nature, 222, 228

Smith D. A., et al., 2008, A\&A, 492, 923

Thorsett S. E., 1991, ApJ, 377, 263

Urama J. O., Link B., Weisberg J. M., 2006, MNRAS, 370, 76

Wang N., Manchester R. N., Zhang J., Wu X. J., Yusup A., Lyne

A. G., Cheng K. S., Chen M. Z., 2001, MNRAS, 328, 855

Wang H. G., et al., 2014, ApJ, 789, 73

Yi S. X., Zhang S. N., 2015, MNRAS, 454, 3674

Yu M., et al., 2013, MNRAS, 429, 688

Yuan J. P., Wang N., Manchester R. N., Liu Z. Y., 2010a, MNRAS, 404, 289

Yuan J. P., Manchester R. N., Wang N., Zhou X., Liu Z. Y., Gao Z. F., 2010b, ApJL, 719, L111

Zhou E. P., Lu J. G., Tong H., Xu R. X. 2014, MNRAS, 443, 2705

This paper has been typeset from a $\mathrm{T}_{\mathrm{E}} \mathrm{X} / \mathrm{LAT}_{\mathrm{E}} \mathrm{X}$ file prepared by the author. 